\title{
Selection of opioids for cancer-related pain using a biomarker: a randomized, multi- institutional, open-label trial (RELIEF study)
}

(1) CrossMark

Hiromichi Matsuoka ${ }^{1,2^{*}}$, Junji Tsurutani ${ }^{3}$, Yasutaka Chiba ${ }^{4}$, Yoshihiko Fujita ${ }^{5}$, Masato Terashima ${ }^{5}$, Takeshi Yoshida ${ }^{1,3}$, Kiyohiro Sakai ${ }^{2}$, Yoichi Otake ${ }^{6}$, Atsuko Koyama ${ }^{1,3}$, Kazuto Nishio ${ }^{5}$ and Kazuhiko Nakagawa ${ }^{3}$

\begin{abstract}
Background: Cancer patients experience pain that has physiological, sensory, affective, cognitive, behavioral, and sociocultural dimensions. Opioids are used in treatment of pain in patients with various types of cancer. We previously showed that the catechol-O-methyltransferase (COMT) genotype is related to the plasma level of morphine and the required dose of morphine in an exploratory prospective study. The findings showed that a group of patients with a GG single nucleotide polymorphism (SNP) rs4680 in COMT required a significantly higher dose of morphine than a non-GG group. A biomarker for selection of opioids for cancer pain relief would be particularly useful clinically, and therefore we have planned a randomized comparative study of morphine and oxycodone, using the COMT rs4680 SNP as a biomarker. This study is aimed at verifying the assumption that patients in the GG group require an increased morphine dose for pain relief.
\end{abstract}

Methods: The RELIEF study is a randomized, multi-institutional, open-label trial with a primary endpoint of the proportion of subjects requiring high-dose opioids, as calculated from the dose of a rescue preparation administered on day 0. Secondary endpoints include the Hospital Anxiety and Depression Scale, Short form McGill Pain Questionnaire-2, European Organization for Research and Treatment of Cancer QLQ-C15-PAL, Pain Catastrophizing Scale, and adverse events, Eligibility criteria are patients with advanced carcinoma with non-daily use of opioids in initial screening for registration; and cancer pain targeted for daily opioid treatment, NSAIDs or acetaminophen, NRS $\geq 3$ (average over $24 \mathrm{~h}$ ), opioid-treatment naive within $30 \mathrm{~h}$, no chemotherapy, radiotherapy, or bisphosphonate administration newly started within 2 weeks, and written informed consent at the time of second registration. Between November 2014 and June 2017, an estimated 110 patients from two sites in Japan were randomized (1:1) to morphine or oxycodone in GG and non-GG groups.

Discussion: A method for selection of appropriate opioids in cancer patients is a high unmet medical need. This study was designed to evaluate the efficacy of different opioids in patients with cancer based on gene polymorphism, as the first potential multi-institutional registration trial to be conducted in cancer patients with pain.

(Continued on next page)

\footnotetext{
* Correspondence: matsuoka_h@med.kindai.ac.jp

'Palliative Care Center, Cancer Center, Kindai University Hospital, 377-2

Ohno-higashi, Osakasayama City, Osaka 589-8511, Japan

2Department of Psychosomatic Medicine, Kindai University Faculty of

Medicine, 377-2 Ohno-higashi, Osakasayama City, Osaka 589-8511, Japan

Full list of author information is available at the end of the article
} 
(Continued from previous page)

Trial registration: UMIN000015579 Date of registration: 4 November 2014. It is updated once every six months, the latest update is 30 June 2017.

Trial status.

The enrollment started in November 2014. At the time of manuscript submission (July 2017), Three-quarters of patients have participated. We thus expect to complete the recruitment by March 2018.

Keywords: Opioid, Biomarker, Cancer pain, Randomized controlled trial

\section{Background}

Opioids are important drugs for cancer pain relief, and definition of an appropriate required dose is needed to provide quick and potent pain relief. The required opioid dose is well known to vary widely among patients, but there have been few studies of biomarkers for the required dose and therapeutic efficacy, or for monitoring of pharmacodynamic effects of opioids. We have previously shown a relationship between cytokines and therapeutic efficacy of morphine [1] and a relationship of catechol-O-methyltransferase (COMT) gene polymorphism with the efficacy and dosage of morphine [2]. These findings emerged in an exploratory prospective study in the Cancer Clinical Research Program funded by a Health and Labor Sciences Research Grant from 2010 to 2012. In particular, we found that patients with a GG single nucleotide polymorphism (SNP) rs4680 in COMT require a significantly higher dose of morphine compared to non-GG patients.

A previous report indicated no relationship between genetic polymorphism and opioid requirement [3], but this report had several limitations, including analysis only in Western patients and uncertainty regarding control subjects, types of pain, use of concomitant medication, presence of symptoms other than pain, and lack of evaluation of pain associated with psychosocial background. Several other studies [4-6] have shown similar results to our preliminary findings, but a recent prospective study showed no relationship between oxycodone dosage and genetic polymorphism [7]. Compared to oxycodone, morphine is available in a greater number of dosage forms, has been used more widely, and is recommended as the first-line drug in many guidelines for cancer pain relief. There is also more information on dyspnea as an adverse effect of morphine. Furthermore, immediate-release morphine reaches a high serum level rapidly and has a shorter sustained duration, and thus is readily used as a rescue drug.

The rationale for the planned study is that morphine and oxycodone are common drugs used for cancer pain, but biomarkers for selection of these drugs, definition of the required dose, and therapeutic efficacy are not available. Based on the above background, there is clearly a practical clinical need for a biomarker in opioid therapy. To identify a biomarker for selection of opioids for cancer pain relief, we planned a randomized comparative study of morphine and oxycodone using the COMT rs4680 SNP as a biomarker. In accordance with criteria described in a previous study of pain [8], the subjects will be patients with a Numerical Rating Scale (NRS) score $\geq 3$ averaged over $24 \mathrm{~h}$. Parallel group comparison will be used for randomization in the study design, so as to generate high-quality evidence.

\section{Methods/design}

Aim, design and setting

Study objectives: A randomized controlled trial (RCT) will be performed with the design shown in Fig. 1. The proportion of subjects requiring high-dose opioids ( $\geq 60 \mathrm{mg} /$ day of morphine or $\geq 40 \mathrm{mg} /$ day of oxycodone administered on day 0 will be calculated from use of immediate-release preparations and compared between the morphine and oxycodone groups using the morphine-equivalent dose in patients with the GG or non-GG COMT rs4680 SNP.

\section{Participants}

Inclusion criteria are 1) patients with advanced malignant tumors, and 2) non-daily use of opioids. At the second registration, the criteria are 1) cancer pain targeted for daily treatment with opioids, NSAIDs or acetaminophen, 2) NRS $\geq 3$ (average over $24 \mathrm{~h}$ ), 3) opioid treatment-naive within $30 \mathrm{~h}, 4)$ no chemotherapy, radiotherapy, or bisphosphonate administration newly started within 2 weeks, and 5) written informed consent.

The exclusion criteria are 1) patients with chronic renal failure (glomerular filtration rate, $30 \mathrm{~mL} / \mathrm{min}$ ), 2) patients with severe hepatic or respiratory failure, and 3) patients deemed ineligible for the study by the study coordinator or a collaborative investigator (ex. neuropathic pain or predominant spontaneous pain only, and have histories of opioid/drug abuse or alcoholism).

\section{Endpoints \\ Primary endpoint}

The primary endpoint is the proportion of subjects requiring high-dose opioids calculated from use of the 


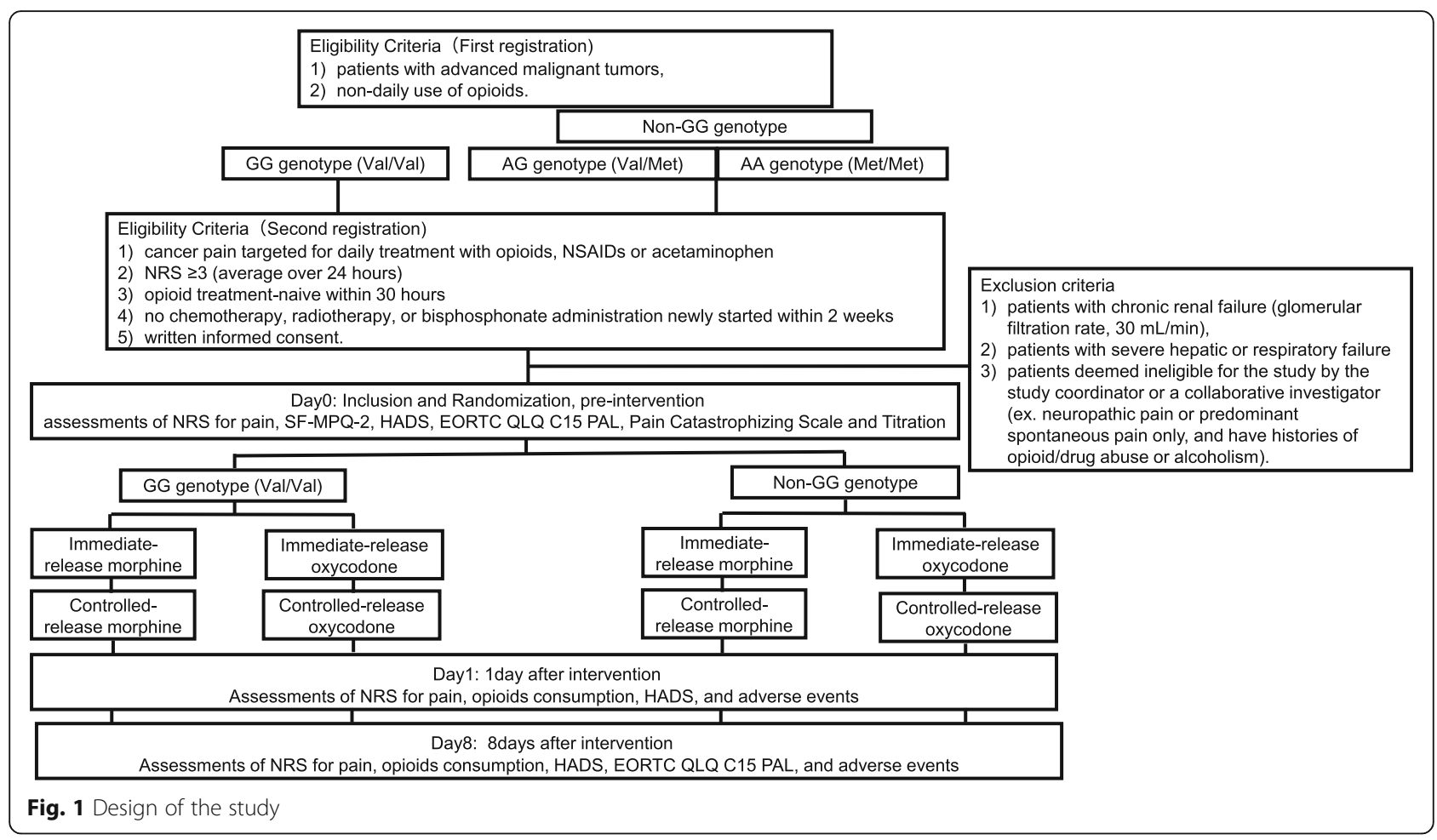

immediate-release preparation on day 0 in a parallel group comparison.

\section{Secondary endpoints}

The secondary endpoints are the Hospital Anxiety and Depression Scale (HADS) score for anxiety and depression; the European Organization for Research and Treatment of Cancer (EORTC) QLQ-C15-PAL for score for QOL; the Short-Form McGill Pain Questionnaire 2 (SFMPQ2) score for pain characterization; and the Pain Catastrophizing Scale (PCS) for estimation of impact on pain prognosis. Adverse events (e.g., constipation, somnolence, nausea, pruritus, ischuria) will be evaluated using the Common Terminology Criteria for Adverse Events (CTCAE) ver. 4.0.

\section{Measurement tools}

Performance status (PS)

The European Cooperative Oncology Group (ECOG) PS system will be used for evaluation of PS by primary physicians [9].

\section{Numerical rating scale (NRS)}

The NRS will be used to evaluate pain for its better validity, sensitivity, and convenience compared to other scales [10] and its widespread use in many clinical studies.

\section{Hospital anxiety and depression scale (HADS)}

The HADS will be used for measurement of psychiatric symptoms (anxiety and depression) of patients with a physical disease. HADS is a screening tool that allows assessment based on a small number of items. Its reliability and validity have been verified internationally $[11,12]$.

\section{European Organization for Research and Treatment of cancer (EORTC) QLQ-C15-PAL}

EORTC QLQ-C15-PAL will be used for evaluation of patient QOL. The reliability and validity of the Japanese version have been confirmed [13].

\section{Short-form McGill pain questionnaire 2 (SF-MPQ-2, Japanese version)}

The SF-MPQ-2 will be used to examine differences in effects due to pain mechanisms. The reliability and validity of the Japanese version have been verified [14].

\section{Pain catastrophizing scale (PCS)}

The severity of cancer-related pain is influenced by engagement of patients in catastrophic thinking, such as "my pain will undoubtedly never improve" [15]. This effect will be measured using the Japanese version of the PCS, for which the validity and reliability have been shown [16]. 
Common terminology criteria for adverse events (CTCAE) The worst grade of an adverse event during the preceding period will be assessed using the CTCAE v.4.0, Japanese Clinical Oncology Group (JCOG) version.

\section{Protocol treatment}

In this prospective clinical study, cancer patients will undergo initial registration and genotyping for SNPs with a Taqman SNP Genotyping Assay (Life Technologies). In the first application of opioid treatment after occurrence of cancer pain, the patients will be divided into a GG group and a non-GG group based on the COMT rs4680 SNP and then undergo second registration, after which the protocol treatment will be started. Each group will be randomized into subgroups that will receive immediate-release morphine ( $T_{\max }$ about $1 \mathrm{~h}$ ) and immediate-release oxycodone $\left(\mathrm{T}_{\max }\right.$ about $\left.2 \mathrm{~h}\right)$, respectively, with doses subjected to titration. Dose titration will be performed to decrease pain by $\geq 33 \%$ on the NRS pain scale, as well as reducing NRS to $\leq 3$. The patients were tested with opioid according to the guideline for titration and following regular dosing (NCCN Guidelines ${ }^{\mathrm{Tm}}$, Adult Cancer Pain) by specialized palliative care doctors. On this step, they explained potential benefits and adverse effects to their patients. Subsequently, the controlled-release opioid will be administered (Fig. 2). In all subgroups, the incidences of subjects requiring a high opioid dose (as a morphine-equivalent dose), psychological tests, and evaluation of QOL are defined as quantitative clinical endpoints to investigate the efficacies of morphine and oxycodone in the GG and non-GG groups. Candidate biomarkers related to onset of adverse effects of opioids will also be measured to examine correlations in an integrated manner. NRS, psychological tests, QOL evaluation, and blood collection will be performed before opioid treatment and on days 1 and 8 after starting treatment. The screening of biomarkers correlated with opioid adverse effects is performed as a subsidiary study. Randomization will be performed on a web page produced by To Field Inc. using the minimization method with modulating factors of age, sex, performance status (PS), and site of pain.

Completion of treatment and use of other drugs: Treatment is completed 8 days after the beginning of the protocol. As supportive therapy, the opioid dose can be increased or reduced as appropriate, based on the decision of a primary physician. Regarding combination therapy, there is no limitation on the use of any other drug.

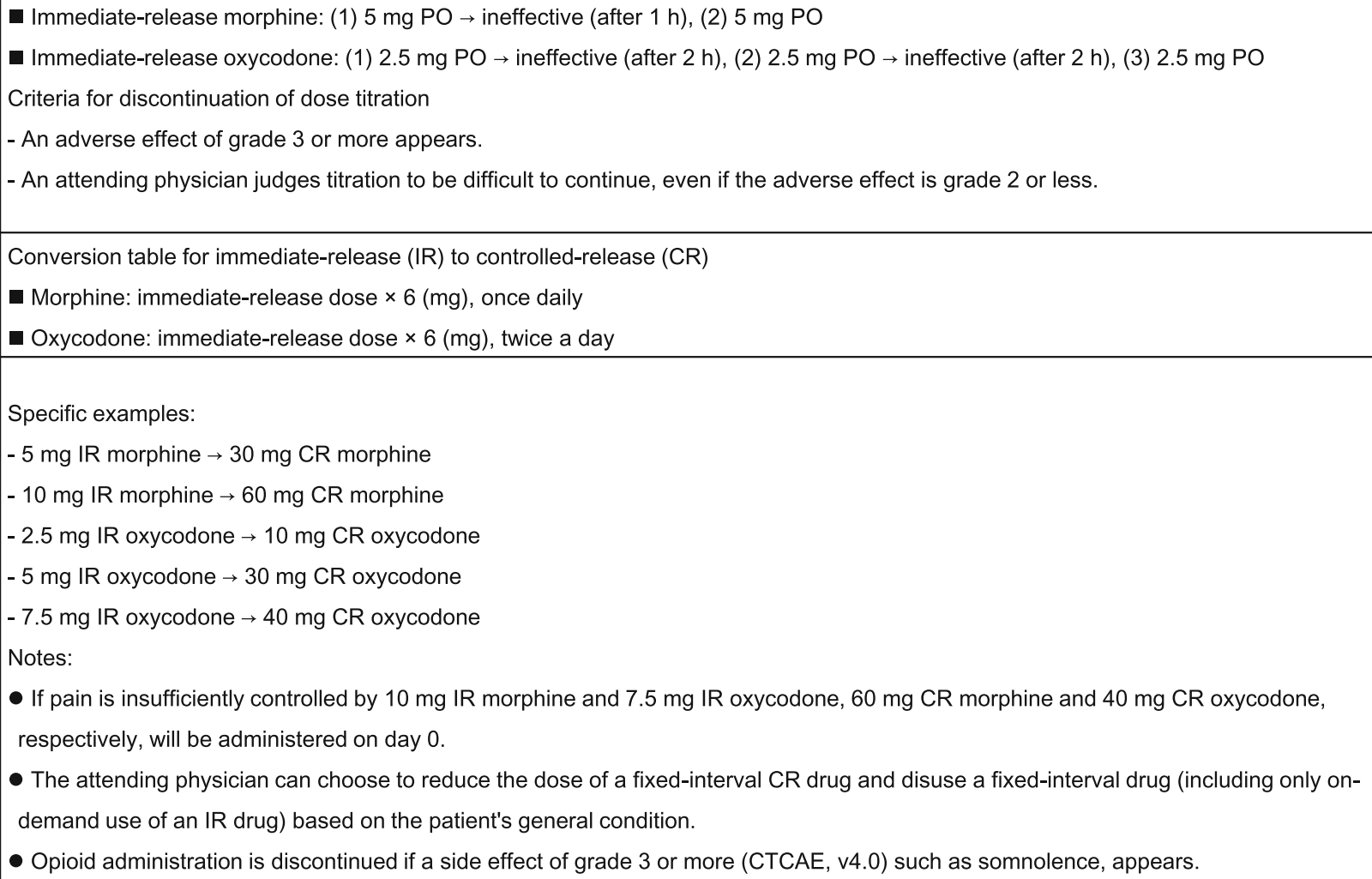

Fig. 2 Methods for dose titration 
Subsidiary biomarker study: This study will include screening for biomarkers correlated with adverse effects of opioids. Therefore, the following items will be examined as potential pharmacological biomarkers: (1) serum chemokine levels, (2) polymorphisms in opioid functionrelated genes, (3) serum glycan analysis, and (4) psychological tests, QOL scale and others. Treatment will be performed in accordance with normal guidelines and will not be changed for this study.

Sample handling: Blood will be collected at initial registration, before starting opioid treatment after second registration, and on days 1 and 8 after starting treatment. Samples will be collected and stored in the laboratory of the Department of Medical Oncology, Kindai University Faculty of Medicine. Samples will be encoded with identification numbers by a manager of personal information at the time of registration.

Ethical issues: All patients are required to provide written informed consent. The study will be performed in accordance with the Declaration of Helsinki and the International Conference on Harmonization and Good Clinical Practice. The protocol has been approved by the Institutional Review Board at each study site.

\section{Statistical analysis}

The null hypothesis is that the proportion of subjects requiring high-dose opioids is equal between the morphine and oxycodone groups for subjects with the GG genotype. This null hypothesis will be evaluated using Fisher's exact test at the one-tailed significance level of $2.5 \%$. The $95 \%$ confidence interval (CI) of the difference in the proportion of subjects requiring high-dose opioids will be calculated as an estimate of the therapeutic efficacy. The proportion of subjects requiring high-dose opioids and the $95 \%$ CI will also be calculated in each group.

Sample size calculation: Our preliminary study [2] indicated that high-dose morphine was required by $36.8 \%$ of GG genotype subjects, and results for 100 subjects in a preceding study at Kindai University and 160 subjects in the series in this study suggested that this proportion was about $46 \%$. On the basis of previous data and discussions at a conference at Kindai University Hospital, about $5 \%$ of these subjects are likely to require highdose oxycodone. Thus, we assumed that $46 \%$ of subjects would require high-dose morphine and 5\% would require high-dose oxycodone in the GG group. Under these assumptions, when we set the one-tailed significance level of $2.5 \%$ and power of $80 \%$, Fisher's exact test requires 31 GG subjects in each drug group; that is, 62 subjects with the GG genotype. Since the GG genotype is estimated to account for around $46 \%$ of the population, the number of registrations required is 135 subjects. Therefore, the target is defined as 140 subjects to allow for dropout and subjects who cannot be analyzed.

\section{Discussion}

This study is the first multicenter RCT of the efficacy of opioids for cancer pain, other than psychogenic pain. We faced three major issues: (i) the heterogeneity of causes of pain, (ii) the absence of a standard of care in this setting, and (iii) the choice of the primary endpoint. Heterogeneity of causes of pains was handled by defining the subjects as patients with any cancer pain except for psychogenic pain. Next, we defined morphine and oxycodone as the standard of care, since these are opioids that are currently used as initial treatment in opioidnaïve patients. We planned to standardize selection for each opioid, because no standard technique has been established for this purpose. Finally, the primary endpoint was defined as the intergroup difference in the number of subjects requiring high-dose opioids based on the COMT rs4680 SNP, with the goal of examining this biomarker for selection of opioids, on the basis of results from previous studies.

The GG COMT rs4680 polymorphism may emerge as a factor predicting therapeutic efficacy, a need for lower doses of oxycodone compared to morphine to exhibit efficacy, and fewer adverse events. Exploratory research may show that this biomarker can also predict side effects. Such findings will contribute to basic understanding of the pharmacological profile and therapeutic efficacy of opioids, as well as establish a practical clinical method for rapid pain relief.

\section{Trial status}

The study protocol was approved by the institutional review board in September 2014. Recruitment started in November 2014 and is currently ongoing.

\section{Abbreviations}

CTCAE: Common Terminology Criteria for Adverse Events; ECOG: European Cooperative Oncology Group; EORTC: European Organization for Research and Treatment; HADS: Hospital Anxiety and Depression Scale; JCOG: Japan Clinical Oncology Group; NCCN: National Comprehensive Cancer Network; NRS: Numerical Rating Scale; PCS: Pain Catastrophizing Scale;

RCT: Randomized controlled trial; SF-MPQ: Short-Form McGill Pain Questionnaire

\section{Acknowledgements}

The authors thank in advance all of the patients, investigators and institutions who will be involved in this study.

\section{Funding}

This research was supported by the following grants: 2014 Health Labour Sciences Research Grant (201438056A), and a 2015-2016 Japan Agency for Medical Research and Development (AMED) award (15ck0106060h0002, 16ck0106060h0003; Innovative Clinical Cancer Research).

Availability of data and materials

Not applicable (data collection is still ongoing).

Authors' contributions

HM participated in design of the study, coordinated study procedures and drafted the manuscript. JT participated in design of the study, and revised the manuscript critically. YC designed the technical treatment strategy and calculated treatment plan parameters. YF, MT and KN will analyze chemokine 
levels, polymorphism of opioid function-related genes, and serum glycans. AK and KN coordinated study procedures and revised the manuscript critically. $T Y, K S$, and YO helped design the study and revised the manuscript critically. All authors read and approved the final manuscript.

\section{Ethics approval and consent to participate}

Institutional review board approvals were obtained from the ethical committee of the Kindai University Faculty of Medicine and Sakai City Medical Center. (reference numbers 26-130 and 1604, respectively). The RELIEF study is published under UMIN000015579. All patients are required to provide written informed consent. The study will be performed in accordance with the Declaration of Helsinki and the International Conference on Harmonization and Good Clinical Practice.

\section{Consent for publication}

Not applicable.

\section{Competing interests}

The authors declare that they have no competing interests.

\section{Publisher's Note}

Springer Nature remains neutral with regard to jurisdictional claims in published maps and institutional affiliations.

\section{Author details}

'Palliative Care Center, Cancer Center, Kindai University Hospital, 377-2 Ohno-higashi, Osakasayama City, Osaka 589-8511, Japan. ${ }^{2}$ Department of Psychosomatic Medicine, Kindai University Faculty of Medicine, 377-2 Ohno-higashi, Osakasayama City, Osaka 589-8511, Japan. ${ }^{3}$ Department of Medical Oncology, Kindai University Faculty of Medicine, 377-2 Ohno-higashi, Osakasayama City, Osaka 589-8511, Japan. ${ }^{4}$ Clinical Research Center, Kindai University Hospital, 377-2 Ohno-higashi, Osakasayama City, Osaka 589-8511, Japan. ${ }^{5}$ Department of Genome Biology, Kindai University Faculty of Medicine, 377-2 Ohno-higashi, Osakasayama City, Osaka 589-8511, Japan. ${ }^{6}$ Department of General Internal Medicine, Sakai City Medical Center, 377-2 Ohno-higashi, Osakasayama City, Osaka 589-8511, Japan.

Received: 17 June 2016 Accepted: 28 September 2017

Published online: 06 October 2017

\section{References}

1. Makimura C, Arao T, Matsuoka H, Takeda M, Kiyota H, Tsurutani J, Fujita Y, Matsumoto K, Kimura H, Otsuka M, Koyama A, Imamura CK, Yamanaka T, Tanaka K, Nishio K, Nakagawa K. Prospective study evaluating the plasma concentrations of twenty-six cytokines and response to morphine treatment in cancer patients. Anticancer Res. 2011;31(12):4561-8.

2. Matsuoka H, Arao T, Makimura C, Takeda M, Kiyota H, Tsurutani J, Fujita Y, Matsumoto K, Kimura H, Otsuka M, Koyama A, Imamura CK, Tanigawara Y, Yamanaka T, Tanaka K, Nishio K, Nakagawa K. Expression changes in arrestin $\beta 1$ and genetic variation in catechol-O-methyltransferase are biomarkers for the response to morphine treatment in cancer patients. Oncol Rep. 2012; 27(5):1393-9.

3. Klepstad P, Fladvad T, Skorpen F, Bjordal K, Caraceni A, Dale O, Davies A, Kloke M, LundstrIN S, Maltoni M, Radbruch L, Sabatowski R, Sigurdardottir V, Strasser F, Fayers PM, Kaasa S. European Palliative Care Research Collaborative (EPCRC); European Association for Palliative Care Research Network. Influence from genetic variability on opioid use for cancer pain: a European genetic association study of 2294 cancer pain patients. Pain. 2011;152(5):1139-45

4. Zubieta JK, Heitzeg MM, Smith YR, Bueller JA, Xu K, Xu Y, Koeppe RA, Stohler CS, Goldman D. COMT Val158Met genotype affects mu-opioid neurotransmitter responses to a pain stressor. Science. 2003;299(5610): 1240-3.

5. Rakvåg TT, Klepstad P, Baar C, Kvam TM, Dale O, Kaasa S, Krokan HE, Skorpen F. The Val158Met polymorphism of the human catechol-Omethyltransferase (COMT) gene may influence morphine requirements in cancer pain patients. Pain. 2005:116(1-2):73-8.

6. Rakvåg TT, Ross JR, Sato H, Skorpen F, Kaasa S, Klepstad P. Genetic variation in the catechol-O-methyltransferase (COMT) gene and morphine requirements in cancer patients with pain. Mol Pain. 2008;4:64.
7. Kambur O, Kaunisto MA, Tikkanen E, Leal SM, Ripatti S, Kalso EA. Effect of catechol-o-methyltransferase-gene (COMT) variants on experimental and acute postoperative pain in 1,000 women undergoing surgery for breast cancer. Anesthesiology. 2013;119(6):1422-33.

8. Kovacs FM, Abraira V, Royuela A, Corcoll J, Alegre L, Tomás M, Mir MA, Cano A, Muriel A, Zamora J, Del Real MT, Gestoso M, Mufraggi N. Spanish Back Pain Research Network. Minimum detectable and minimal clinically important changes for pain in patients with nonspecific neck pain. BMC Musculoskelet Disord. 2008:9:43.

9. Oken MM, Creech RH, Tormey DC, Horton J, Davis TE, McFadden ET, et al. Toxicity and response criteria of the Eastern Cooperative Oncology Group. Am J Clin Oncol. 1982;5:649-55.

10. Caraceni A, Cherny N, Fainsinger R, Kaasa S, Poulain P, Radbruch L, De Conno F. Pain measurement tools and methods in clinical research in palliative care: recommendations of an Expert Working Group of the European Association of Palliative Care. J Pain Symptom Manag. 2002:23:239-55.

11. Zigmond AS, Snaith RP. The hospital anxiety and depression scale. Acta Psychiatr Scand. 1983;67(6):361-70.

12. Kugaya A, Akechi T, Okuyama T, Okamura H, Uchitomi Y. Screening for pyschological distress in Japanese cancer patients. Jpn J Clin Oncol. 1998;28(5):333-8.

13. Miyazaki K, Suzukamo Y, Shimozuma K, Nakayama T. Verification of the psychometric properties of the Japanese version of the European Organization for Research and Treatment of Cancer Quality of Life Questionnaire Core 15 palliative (EORTC QLQ-C15-PAL). Qual Life Res. 2012;21(2):335-40.

14. Maruo T, Nakae A, Maeda L, Shi K, Takahashi K, Morris S, Hosomi K, Kanatani $H$, Matsuzaki T, Saitoh Y. Validity, reliability, and assessment sensitivity of the Japanese version of the short-form McGill Pain Questionnaire 2 in Japanese patients with neuropathic and non-neuropathic pain. Pain Med. 2014;15(11):1930-7.

15. Utne I, Miaskowski C, Bjordal K, Paul SM, Jakobsen G, Rustøen T. Differences in the use of pain coping strategies between oncology inpatients with mild vs. moderate to severe pain. J Pain Symptom Manag. 2009:38(5):717-26.

16. Matsuoka H, Sakano Y. Assessment of cognitive aspect of pain: Development, reliability, and validation of Japanese version of pain catastrophizing scale. Jpn J Psychosoma Med. 2007;47:95-102.

\section{Submit your next manuscript to BioMed Central and we will help you at every step:}

- We accept pre-submission inquiries

- Our selector tool helps you to find the most relevant journal

- We provide round the clock customer support

- Convenient online submission

- Thorough peer review

- Inclusion in PubMed and all major indexing services

- Maximum visibility for your research

Submit your manuscript at www.biomedcentral.com/submit
) Biomed Central 